\title{
Changes on lysosomal compartment during PMA-induced differentiation of THP-1 monocytic cells: Influence of type I and type IV collagens
}

\author{
Alessandra Spano ${ }^{1}$, Sergio Barni ${ }^{2}$, Vittorio Bertone ${ }^{2}$, Luigi Sciola ${ }^{1}$ \\ ${ }^{1}$ Dipartimento di Scienze Biomediche, Università di Sassari, Sassari, Italy \\ ${ }^{2}$ Dipartimento di Biologia e Biotecnologie "Lazzaro Spallanzani”, Università di Pavia, Pavia, Italy \\ Email:sciola@uniss.it
}

Received 26 May 2013; revised 30 June 2013; accepted 16 July 2013

Copyright (C) 2013 Alessandra Spano et al. This is an open access article distributed under the Creative Commons Attribution License, which permits unrestricted use, distribution, and reproduction in any medium, provided the original work is properly cited.

\section{ABSTRACT}

In this work, the influence of different substrate adhesion during phorbol-12-myristate-13-acetate (PMA)induced differentiation of THP-1 monocytic cell line was studied. In particular, by morphocytochemical and cytometric approaches, the influence of type I and type IV collagens in an experimental model representative of three phases (initial, intermediate and terminal) of monocyte-macrophage transition was analyzed. The cells in these three phases of differentiation were obtained by using 6, 30 e 60 nM PMA. In this experimental model, referring to adhesion to glass as control, by using the azo-dye coupling method, we have considered the analysis of Acid Phosphatase (AcP) activity as a marker of differentiated status expression, in relation to the acquisition of macrophagic phenotype. Endosomal/lysosomal system was further characterized by taking into account the uptake of fluorescent probe LysoTracker Red. Fluorochromization in the various experimental conditions was analyzed morphologically (fluorescence microscopy) and quantitatively (static cytometry). Data related to lysosome compartment were integrated, from a cytokinetic point of view, by flow cytometry measurements of DNA/protein content. Our results have indicated that type I and type IV collagens were able to influence, with respect to glass adhesion, various differentiation phases. Type I collagen showed the higher effects in the condition of high differentiation (60 $\mathrm{nM}$ PMA), causing an increase in AcP activity and lysosomal system. Type IV collagen, besides determining effects on lysosomal compartment of intermediate and terminally differentiated cells, influenced mainly proliferative activity of cells with initial differentiation level (6 nM PMA).
Keywords: THP-1 Cells; Differentiation; Type I and IV Collagens; Acid Phosphatase; Lysosomes

\section{INTRODUCTION}

Comparison between human blood monocytes and derived macrophages obtained after differentiation has demonstrated a modulation of phenotype [1-6]. Some works [7] have shown that in vitro maturation of blood monocytes into macrophages could be used as a model for studying the in vivo phenomenon. Monocyte-derived macrophages generally express surface markers not found on their monocyte precursors $[2,4,6,7]$. The increase in the appearance of macrophage specific markers/activities is associated with a decrease in certain monocyte characteristics. Various morpho-functional features can be considered to reach the completion of differentiated status. Macrophages can be characterized in unstimulated, tissue-resident or fully activated stages. Activated macrophages characteristically demonstrate increased membrane ruffling, increased adhesion and spreading, stimulation of DNA synthesis, modified monokine secretion, increased lysosomal enzyme levels, changes in phagocytic activity and increased bactericidal/tumoricidal activity [8]. Phagocytosis is prominent in macrophages and crucial for their functions.

For studying these and other functions, monocytes from peripheral venous blood or cell lines are usually employed. Differentiation protocols have been developed treating monocytic cell lines such as U937, HL-60 or THP-1 cells with stimuli such as phorbol-12-myristate13-acetate (PMA) or 1,25-dihydroxy vitamin D3 [9-12]. The phenotype of the macrophages in these protocols varies with the differentiation treatment and duration, reflecting differences in gene transcription [13,14]. PMA treatment, which activates protein kinase $\mathrm{C}$, also induces 
a greater degree of differentiation in THP-1 cells as reflected by increased adherence and expression of surface markers associated with macrophage differentiation [15, 16]. However, it is unclear how the differentiation state of PMA-treated THP-1 cells compares with that of tissue macrophages or monocyte-derived macrophages, a well recognized model of differentiated tissue macrophages [17]. In contrast to native human monocytes, a cell line such as THP-1 offers the additional advantage of a population that markedly facilitates morpho-functional studies. Under the influence of the phorbol ester, PMA, THP-1 cells stop proliferating and differentiate into macrophage-like cells $[14,18]$. The differentiation of the THP-1 cells is associated with a strong change in cell morphology. After the initiation of this differentiation process, cells acquire a variety of shapes, their nucleus becomes more irregular, and many phagocytic vacuoles can be recognized in their cytoplasm. This differentiation process is also associated with an increased adherence to tissue culture plastic. Furthermore, differentiated THP-1 cells show changes in forward scatter characteristics upon flow cytometry, which indicate a change of cell volume during differentiation [18].

The use of in vitro cell models of monocyte-macrophage differentiation demonstrates that this process is influenced by several factors: in monocytes from peripheral blood, beside serum as a differentiation stimulus, differentiation is also dependent on other parameters. Cell purity of the monocyte preparation, which is dependent on the separation technique, is one crucial aspect because contaminating lymphocytes can modulate the differentiation process. When monocytic cell lines are used, it must be conveniently considered cell population heterogeneity and its interaction with serum factors [16]. Another problem is the choice of the culture substrate. Differentiating monocytes become adherent cells, and various functions are modulated by cell adhesion. De Fougerolles et al. [19] have described distinct morphology and antigen expression dependent on the culture substrate. We have previously demonstrated, in the consolidated THP-1 cell model, that treatment for $72 \mathrm{~h}$ with concentrations ranging from 6 to $60 \mathrm{nM}$ of the phorbol ester PMA was able to induce a gradual progression of phenotype from monocyte to terminally differentiated macrophage that appeared to be related to the progressive increase of the adhesive capacity [18]. In particular, three concentrations of 6,30 and $60 \mathrm{nM}$ permit to identify specific stages of monocyte towards macrophage path coinciding with initial, intermediate and terminal differentiation, respectively. In the present study, this in vitro model system was employed, by using the same PMA doses, to examine the influence of extracellular matrix (ECM) proteins, such as type I and type IV collagens on monocyte differentiation and activation. Analy- sis of macrophage specific activities demonstrated that monocyte-macrophage differentiation in vitro is influenced by ECM proteins. In this experimental model, we have considered the detection of acid phosphatase (AcP) activity as a marker of differentiated status expression in relation to the acquisition of macrophagic phenotype. Endosomal/lysosomal systems are essential for macrophage functions. AcP is a hydrolase contained inside this system [20,21] and is considered to provide cytochemical criteria for the evaluation of macrophage activation $[22,23]$. From a methodological point of view, AcP activity was determined by optical cytochemical reactions. The data obtained were related to the general distribution of endosomal/lysosomal systems monitored by using LysoTracker Red (LT) probe, a fluorophore linked to a weak base, only partially protonated at neutral $\mathrm{pH}$, freely permeant to cell membranes, and selectively accumulated in cellular compartments with low internal pH. LT can be used to label and then investigate and track acidic organelles in living cells [24]. To obtain quantitative data on total cellular fluorescence, LT-stained cells were submitted to measurements by static cytometry. Cytokinetic data, evidence on changes of cellular size during PMA treatment, also in relation to the ability to spread on different substrata, were obtained by analyzing flow cytometry cytograms, after fluorochromization of DNA and total proteins.

\section{MATERIALS AND METHODS}

\subsection{Cell Culture and PMA Treatment}

THP-1 cells, derived from a human monocytic leukaemia, were grown in RPMI 1640 supplemented with $15 \%$ fetal calf serum. The cultures were maintained at $37^{\circ} \mathrm{C}$ in a humidified atmosphere $\left(95 \%\right.$ air $\left./ 5 \% \mathrm{CO}_{2}\right)$ at a density of $0.8-1 \times 10^{5}$ cells $/ \mathrm{ml}$, as a stationary suspension culture. For microscopy analyses, THP-1 cells were grown, on glass coverslips, in multiwell plates $\left(0.8-1.0 \times 10^{5}\right.$ cells/ $\mathrm{cm}^{2}$ ).

Activation of the monocytic THP-1 cell line was obtained by incubation with different concentrations $(6,30$, and $60 \mathrm{nM}$ in complete medium) of the phorbol ester PMA (Phorbol 12-myristate 13-acetate) (SIGMA, St. Louis, MO, USA). After treatment for $72 \mathrm{~h}$, the cells were induced to adhere to the growth substrate and to differentiate. PMA was changed every day and, in relation to the different concentrations, the cells progressively acquired the morphofunctional features related to the level of differentiation.

\subsection{Cell Adhesion}

Glass coverslips non-coated or coated with type I, from calf skin, or type IV, from human nourishment organ, 
collagens (SIGMA, St. Louis, MO, USA) $(0.1 \%$ in $0.1 \mathrm{M}$ acetic acid; $10 \mu \mathrm{g} / \mathrm{ml}$ ) were used. After collagen solution addition, glass coverslips were allowed to dry in a laminar flow hood. THP-1 cells were seeded $\left(2 \times 10^{6}\right.$ cells $\left./ \mathrm{ml}\right)$ in wells containing coverslips (coated and non-coated) in the presence of PMA at different concentrations. After 24 $\mathrm{h}$, non adherent monocytes were removed by gently washing with PBS. As internal control, THP-1 cells, maintained as stationary suspension, were used and only for microscopic analysis and static cytometry, the cells were cytocentrifuged $(200 \times \mathrm{g}$ for $10 \mathrm{~min})$ on glass coverslips previously coated with Poly-L-Lysine Hydrobromide (SIGMA, St. Louis, MO, USA) (300 $\mu \mathrm{g} / \mathrm{ml}$ in PBS).

\subsection{Flow Cytometry of DNA/Protein Relative Content}

Cell measurements were performed by means of a PARTEC PAS II Flow cytometer (PARTEC, Münster, Germany). THP-1 cells, maintained as stationary suspension in T-25 $\mathrm{cm}^{2}$ culture flasks, were collected by centrifugation (100 g for $10 \mathrm{~min})$, while PMA-treated adherent cells $\left(2.5-3.0 \times 10^{6}\right.$ cells $\left./ \mathrm{T}-25 \mathrm{~cm}^{2}\right)$ were collected by trypsinization $(0.25 \%$ trypsin in PBS). After washing in PBS, the cells were fixed in $70 \%$ ethanol at $4^{\circ} \mathrm{C}$ for 30 min. The measurements of DNA/protein relative content were performed after fluorochromization $(45 \mathrm{~min}$ at room temperature) with $8 \mu \mathrm{M}$ DAPI (4',6-diamidino-2phenylindole) (SIGMA, St. Louis, MO, USA) and $50 \mu \mathrm{M}$ SR 101 (Sulforhodamine 101) (SIGMA, St. Louis, MO, USA) in $0.1 \mathrm{M}$ Tris-HCl buffer $\mathrm{pH} 7.5$, respectively. The cells were then filtered with a nylon filter $($ mesh $=50 \mu \mathrm{m})$ in order to remove cellular aggregates. Cell concentration was adjusted to approximately $4-6 \times 10^{5}$ cells $/ \mathrm{ml}$, in order to minimize the errors during the flow measurements. The instrument equipped with an $\mathrm{Hg}$ lamp was used in the optic configuration for UV ( $\lambda$ exc. $=350 \mathrm{~nm})$, for the simultaneous DAPI $(\lambda \mathrm{em} .=435 \mathrm{~nm})$ and SR 101 $(\lambda \mathrm{em} .=630 \mathrm{~nm})$ excitation. The data were collected in DNA/protein content cytograms. The comparison between the different experimental conditions was carried out by the Student's $t$-test, and the differences were considered statistically significant with $p<0.05$.

\subsection{Lysosome Compartment Fluorochromization and Static Cytofluorometry}

\subsubsection{Fluorescence Microscopy}

Lysosome compartment was visualized in vital cells by incubating the cultures in the presence of LysoTraker Red (Molecular Probes, Eugene, OR). For this vital staining, cells were incubated with the probe $(100 \mathrm{nM})$ for $20 \mathrm{~min}$ at $37^{\circ} \mathrm{C}$. Nuclear counterstaining was performed by means of Hoechst 33342 (SIGMA, St. Louis,
MO, USA) $(2 \mu \mathrm{g} / \mathrm{ml}$ in PBS) for $10 \mathrm{~min}$ at room temperature. After being washed in PBS, coverslips were mounted in culture medium. Observation was performed by epifluorescence with a NIKON Eclipse 600 microscope (NIKON, Kanagawa, Japan) equipped with a 100$\mathrm{W}$ mercury lamp. The following conditions were used: 330 - $380 \mathrm{~nm}$ excitation filter (excf), $400 \mathrm{~nm}$ dichroic mirror $(\mathrm{dm})$ and $420 \mathrm{~nm}$ barrier filter (bf) for Hoechst 33342 and $540 \mathrm{~nm}$ excf, $580 \mathrm{~nm} \mathrm{dm}$ and $590 \mathrm{~nm}$ bf for LysoTracker Red.

\subsubsection{Static Cytofluorometry}

Fluorescence intensity per cell was evaluated after lysosome/phagosome staining, by measuring fluorescence emitted by each single cell. For this analysis, a NIKON P II (NIKON, Kanagawa, Japan) cytoflurometer was used. Within the cytometric apparatus, an appropriate system of diaphragms permitted selection of single cells and fluorescence was quantitatively measured after automatic subtraction of background brightness. The quantitative data, expressed as arbitrary units (a.u.), were collected in frequency histograms of fluorescence intensity. For each experimental condition, three representative slides were examined, and at least 250 cells per slide were measured.

\subsection{Cytochemical Reaction for Acid Phosphatase Detection}

Cell suspension (monocytes) or adherent cells (macrophages at different level of differentiation) were fixed in $2 \%$ paraformaldheyde (PFA) in PBS for $30 \mathrm{~min}$ at room temperature. After fixation, specimens with adherent cells were washed twice in PBS, once again in sterile water and then air-dried. For monocytes, after washes in PBS, the samples were cytocentrifuged, rapidly washed in sterile water and coverslips were air-dried. For detection of acid phosphatase, the azo-dye coupling method was used. The enzyme expression was demonstrated by the hexasotised pararosanilin/naphtol-ASBI-phosphate method of Barka \& Anderson [25]. The negative reaction control was performed by the addition of sodium fluoride to the staining solution. The slides were scored with a bright-field Zeiss Axioskop Plus microscope equipped with Differential Interference Contrast (DIC) (Zeiss, Jena, Germany). The evaluation of cytochemically positive cells was calculated as the percentage of a total number (about 500) for each experimental condition, in different microscopic fields; the data are expressed as mean \pm SEM. Statistical analysis was assessed by Kruskal-Wallis test in the different culture conditions by comparing the effects of PMA concentrations in the same cell adhesion substrate and the effects of the different substrate at the same PMA concentration. Results were considered to be significant with $p<0.05$. 


\subsection{Imaging Methods}

In the different experimental conditions, microscopic fields have been acquired by means of Color View III CCD camera (resolution up to $2576 \times 1932$ imaging pixel in color mode) and analyzed by means of AnalySIS image analysis system (Soft Imaging System- $\mathrm{GmbH}$, Münster-Germany).

\section{RESULTS}

\subsection{Cytokinetic Activity and Analysis of Protein Content during the Cell Differentiation}

The analysis of cytofluorometric measurements of the relative DNA and protein content correlation (Figure 1), after 6, 30, and $60 \mathrm{nM}$ PMA treatment, allowed us to obtain some information on the cytokinetic changes during the differentiation of the THP-1 monocytic cell line in macrophages, in absence (adhesion to glass: Figures 1 (b)-(d)) or presence of type I (Figures 1(e)-(g)) and type IV (Figures 1(h)-(j)) collagens. As far PMA treatment is concerned, the comparison between cytograms of untreated THP-1 cells (monocytes: Figure 1(a)) and during treatment for $72 \mathrm{~h}$ with the phorbol ester (Figures 1(b)(d) shows a progressive decrease of cell proliferation activity as revealed by the fall down of number of cells with interploid DNA values. From a cytokinetic point of view, type I and IV collagen adhesion tend to determine, especially after $6 \mathrm{nM}$ PMA (Figures 1(e), (h)), the increase in cell number with intermediate DNA content, with respect to glass adhesion (Figure 1(b)). Others aspects were also suggested by the analysis of patterns of biparametric DNA/protein cytograms: in monocytic line condition (Figure 1(a)), the cells showed homogeneous values of protein content coinciding with the $2 \mathrm{c}$ and $4 \mathrm{c}$ DNA values and high number of cells with intermediate values of DNA content. After PMA stimulation, especially for $60 \mathrm{nM}$ PMA (advanced cell differentiation condition: Figure 1(d)), the two cell populations showed an evident increase in protein content, characterized by a high degree of value dispersion, indicating wide cell heterogeneity with the presence of $2 \mathrm{c}$ and $4 \mathrm{c}$ cells with doubled mass. Cytogram analysis of 6 nM PMA-treated cultures adherent on type I (Figure 1(e)) or type IV (Figure 1(h)) collagen-coated substrate, com- pared to those relative to glass adherent cultures (Figure 1(b)), did not show a significant difference in the higher value of proteic content in case of $4 \mathrm{c}$ subpopulations. Moreover, the trend to a moderate increase in proteic mass (160 vs 140 arbitrary units: a.u.) of $2 \mathrm{c}$ populations is observed. After $30 \mathrm{nM}$ PMA, a sharp change on DNA/protein ratio distribution of $4 \mathrm{c}$ sub-population in cultures adherent on type I collagen (Figure 1(f)) was revealed: the mean value of proteic content is settled around 130 a.u., while in glass adhesion condition, for the same PMA stimula- tion (Figure 1(c)), the mean value of ordinate axis is around 110 a.u. The $2 \mathrm{c}$ sub-population did not show significant overall changes, even if an increase in the modal value of proteic content values is noticed (Figure 1(f)). As far as $30 \mathrm{nM}$ PMA stimulated cultures adherent to type IV collagen are concerned, $2 \mathrm{c}$ and $4 \mathrm{c}$ cells showed a higher dimensional homogeneity and a reduced scattering of proteic content (Figure 1(i)). After $60 \mathrm{nM}$ PMA stimulation, in cells adherent to type I collagen (Figure 1(g)), the 2c subpopulation was noticeable increased in percentage and showed a high dispersion of protein content: the highest value was around 220 a.u. This was in part also observed after adhesion to type IV collagen (Figure 1(j)); moreover, in this condition, depletion of $4 \mathrm{c}$ subpopulation (Figure 1(j)) is lesser than that observed, for the same subpopulation, after type I collagen adhesion (Figure 1(g)). In the three types of adhesion substrate, intermediate DNA values were undetectable (Figures 1(d), (g), (j)).

\subsection{Lysosome Compartment Fluorochromization and Static Cytofluorometric Measurements}

In condition of stationary suspension, fluorescence microscopy images (Figure 2(a)) showed a limited fluorescence in all the cells, distributed as spots and in a relatively uniform way, in the cytoplasm of cells with rounding shape and a sharp dimension homogeneity. This homogeneity is confirmed not only by cytogram of DNA/ protein relative content (Figure 1(a)), but also by the distribution of LysoTracker Red fluorescence intensity values in this experimental condition (Figure 3(a)). Nevertheless, the comparison between the three adhesion conditions, as also confirmed by static cytofluorometry analysis, did not show significant differences in LysoTracker Red staining distribution (Figures 3(b), (e), (h)). At $30 \mathrm{nM}$ PMA dose, the increase in number and fluorescent spot intensity is related to their prevailingly localization at juxta-nuclear area and to evident changes of both cytoplasmic and nuclear morphology; in general, a marked cell heterogeneity is observed (Figures 2(c), (f), (i)).

This is partly confirmed by the analysis of histograms relative to LysoTracker Red fluorescence intensity, in the conditions of adhesion to glass (Figure 3(c)), type I collagen (Figure 3(f)) and type IV collagen (Figure 3(i)), which appears to show a some dispersion and, for type IV collagen, some high fluorescence values, relative to polyploid cells with high dimension (Figure 3(i) and Figure 6).

After $60 \mathrm{nM}$ PMA, both fluorescence images (Figures 2(d), (g), (j)) and histograms relative to fluorescence measurements (Figures 3(d), (g), (j)) indicated that, for this dose of phorbol ester, there is a larger and intense 


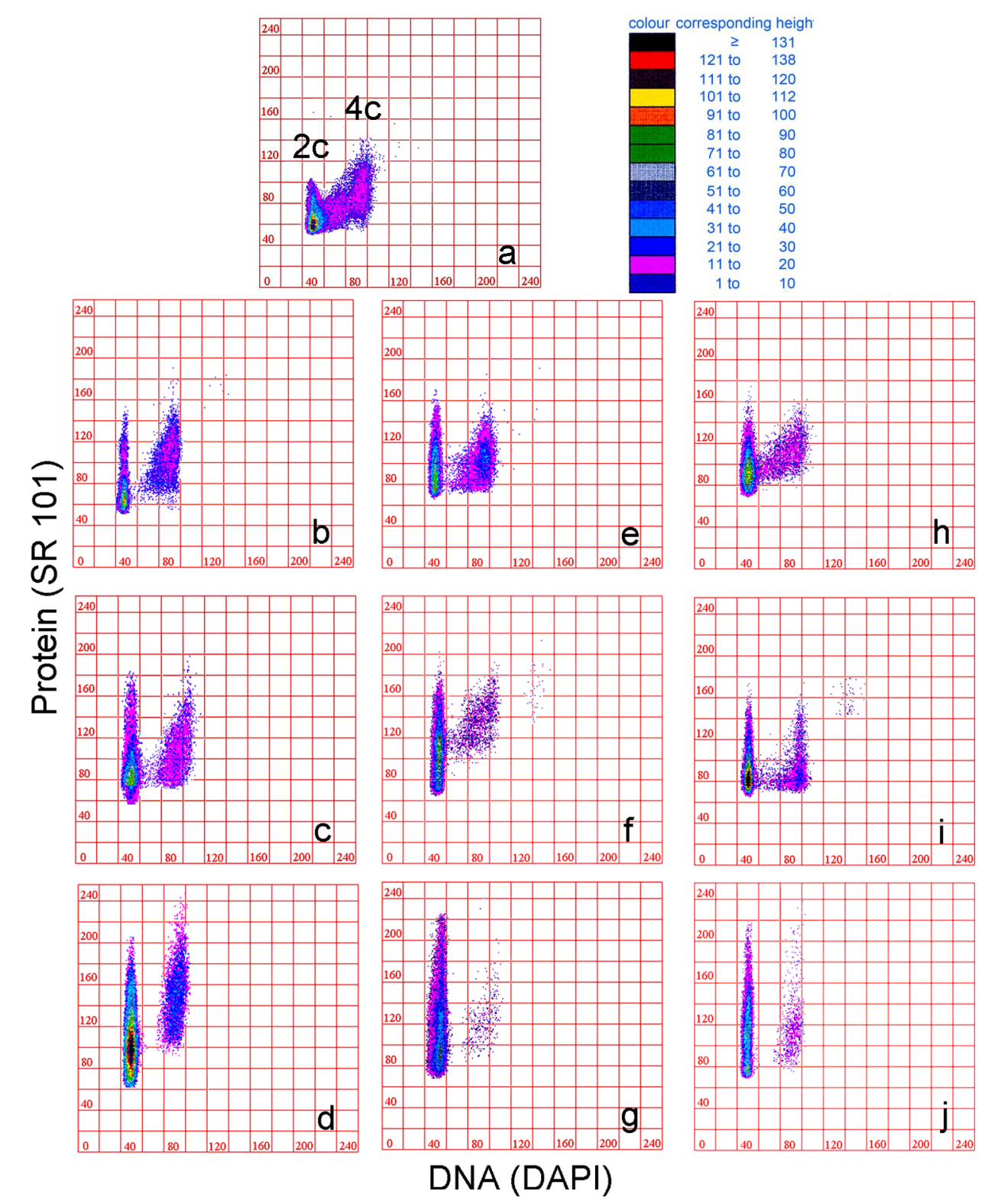

Figure 1. Cytograms of DNA versus protein cell contents (flow cytometry after DAPI/SR101 fluorochromization) in THP-1 cell line as monocytic suspension (a) and after adhesion (PMA stimulation) to glass (b-d), to type I (e-g) and type IV (h-j) collagens. PMA concentration: (a) 0 $\mathrm{nM}$; (b, e, h) $6 \mathrm{nM}$; (c, f, i) $30 \mathrm{nM}$; (d, g, j) $60 \mathrm{nM}$. All cytograms are representative of one of three experiments.

presence of fluorescent spots. As indicated by images (Figure 2(g)) and cytofluorometric measurements (Figure $3(\mathrm{~g})$ ), these spots showed the higher expression level in the case of adhesion to type I collagen.

\subsection{Acid Phosphatase Activity Detection}

Acid phosphatase activity of THP-1 cells in the different experimental conditions was found to vary significantly. The increased intensity of the cytochemical reaction for this lysosomal enzyme appeared to be related to the increase in PMA concentration and also dependent on the nature of the cell adhesion substrate used for the culture (Figure 4). The analysis of images obtained after conventional optic and interference contrast microscopy combination permitted to observe a limited cytochemical reaction development in $6 \mathrm{nM}$ PMA stimulated cultures adherent to glass (Figure 4(a)) or to type I collagen (Figure 4(c)) and type IV collagen (Figure 4(e)).

On contrary, the highest frequency of cytochemically labelled cells and the more intense reaction staining were reached after $60 \mathrm{nM}$ PMA in the presence of type I collagen as cell adhesion substrate (Figure 4(d)). In general, 

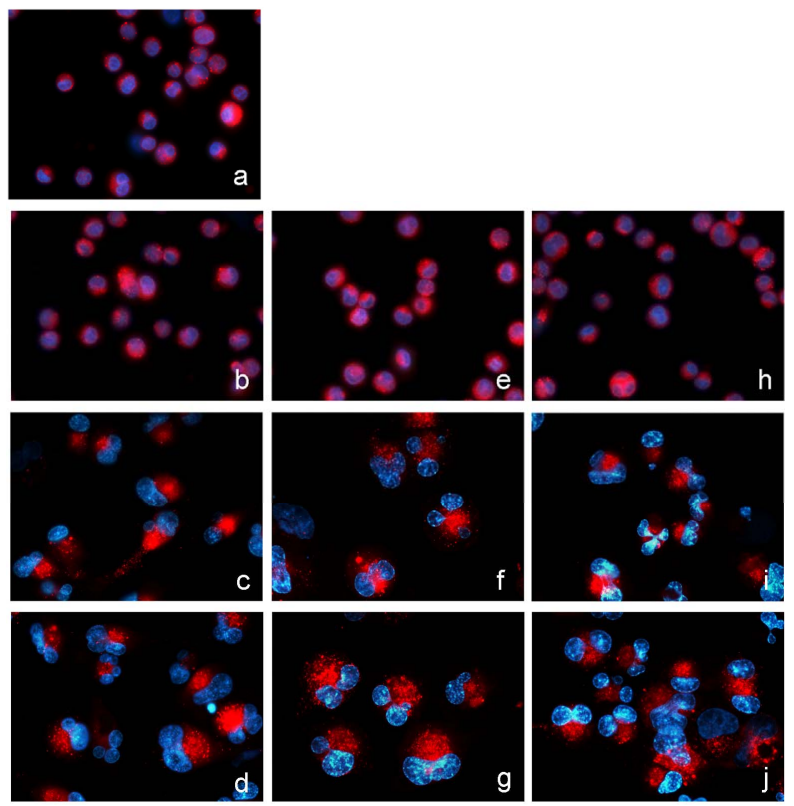

Figure 2. Fluorescence microscopy, after LysoTracker Red fluorochromization, of THP-1 cell line as monocytic suspension (a) and after adhesion (PMA stimulation) to glass (b-d), to type I (e-g) and type IV (h-j) collagens. Nuclei are counterstained with Hoechst 33342. PMA concentration: (a) $0 \mathrm{nM}$; (b, e, h) $6 \mathrm{nM}$; (c, f, i) $30 \mathrm{nM}$; (d, g, j) $60 \mathrm{nM}$. (objective $50 \times$ ) Scale bar: $5 \mu$.

for all PMA doses, significant differences by comparing cultures adherent to type I and type IV collagens were observed (Figure 5). For type I collagen, the frequency of positive cells to cytochemical reaction was always higher than that observed for type IV collagen (Figure $5)$.

The same situation can be noticed by comparing cells adherent to both collagen types and to glass (Figure 5). From a morphological point of view, cytochemical patterns were correlated with the presence of both lysosomes and phagosomes, frequently concentrated in the iuxta-nuclear region. This was particularly evident especially in cells with higher size and multilobated nucleus (Figure 6).

\section{DISCUSSION}

Factors leading to monocyte-macrophage differentiation and activation remain partially uncharacterized. In this work, we have investigated the contribution of interaction with type I and type IV collagens on modulation of differentiation of THP-1 monocytic cells during PMA stimulation.

The interaction with matrix components can contribute to regulating monocyte and macrophage behaviour, as suggested by previous studies $[19,26]$ examining the role of signals mediated through matrix receptors, such as integrins $[27,28]$. For many cell types, integrin-mediated adhesion to matrix is required to proceed normally through the G1 phase of the cell cycle [28,29]. Moreover, studies performed on biochemical basis have demonstrated the importance of collagen in favoring the process of THP-1 cell differentiation during adhesion, by way of involvement of several modulating molecules, among which interleukin family is included [30].

In our experimental model, THP-1 cells undergo, simultaneously, the consequences of both PMA and substrate interaction, represented, alternatively, by the two kinds of collagen. As far as the effects of only PMA stimulation (adhesion to glass) are concerned, this phorbol ester causes cell cycle slow-down/arrest: such effects appear to be dose-dependent [16,18]. Antiproliferative and cyto-differentiative activity of PMA on THP-1 cells could implicate changes in expression of proto-oncogenes involved in the modulation of equilibrium status between mitotic capability and differentiated status of the cells [31]. Thus, this experimental biological situation can follow the "principle of inverse correlation" between cell proliferation and differentiation through a complex system of regulation of the cell cycle [32]. As far as the effects of extracellular matrix components are concerned, in our experimental model, cell adhesion to a solid substrate of collagen, especially for type IV collagen with respect to type I collagen, caused a resumption of cell proliferation, as suggested by the increase in $2 \mathrm{c}-4 \mathrm{c}$ values. This datum is in agreement with previous studies on other cell models [33] and, in our experiments, it is particularly evident especially for $6 \mathrm{nM}$ PMA stimulated cultures, while the effect is reduced after $30 \mathrm{nM}$ PMA or missing after $60 \mathrm{nM}$ PMA treatment. From these findings, it emerged that adhesion on both collagen types caused different effects in cells that have reached different levels of differentiation: cells showing some proliferative activity (after $6 \mathrm{nM}$ PMA stimulation) are partly induced to divide, while cells with a high differentiative level are not stimulated to proliferation; this contributes to the percentage increase in $2 \mathrm{c}$ sub-population, rationally constituted by more differentiated cells. These cytokinetic features are correlated with the acquisition of a strong adhesive capability towards the substrate, in which collagen-binding integrins could play a role [27]. Adhesive events may allow the stabilization of integrins at cell surfaces and could enable signaling events responsible for acquiring the resident status of macrophage and for its activation state.

By using other cell model, some authors [34] indicated that culturing on type I collagen doubled the number of differentiated monocytes at 24 hours and was a stronger stimulus for differentiation than phorbol myristate acetate, a known inducer of monocyte differentiation. As far as type IV collagen is concerned, it is well known for its role on the organization of basement membrane in which 

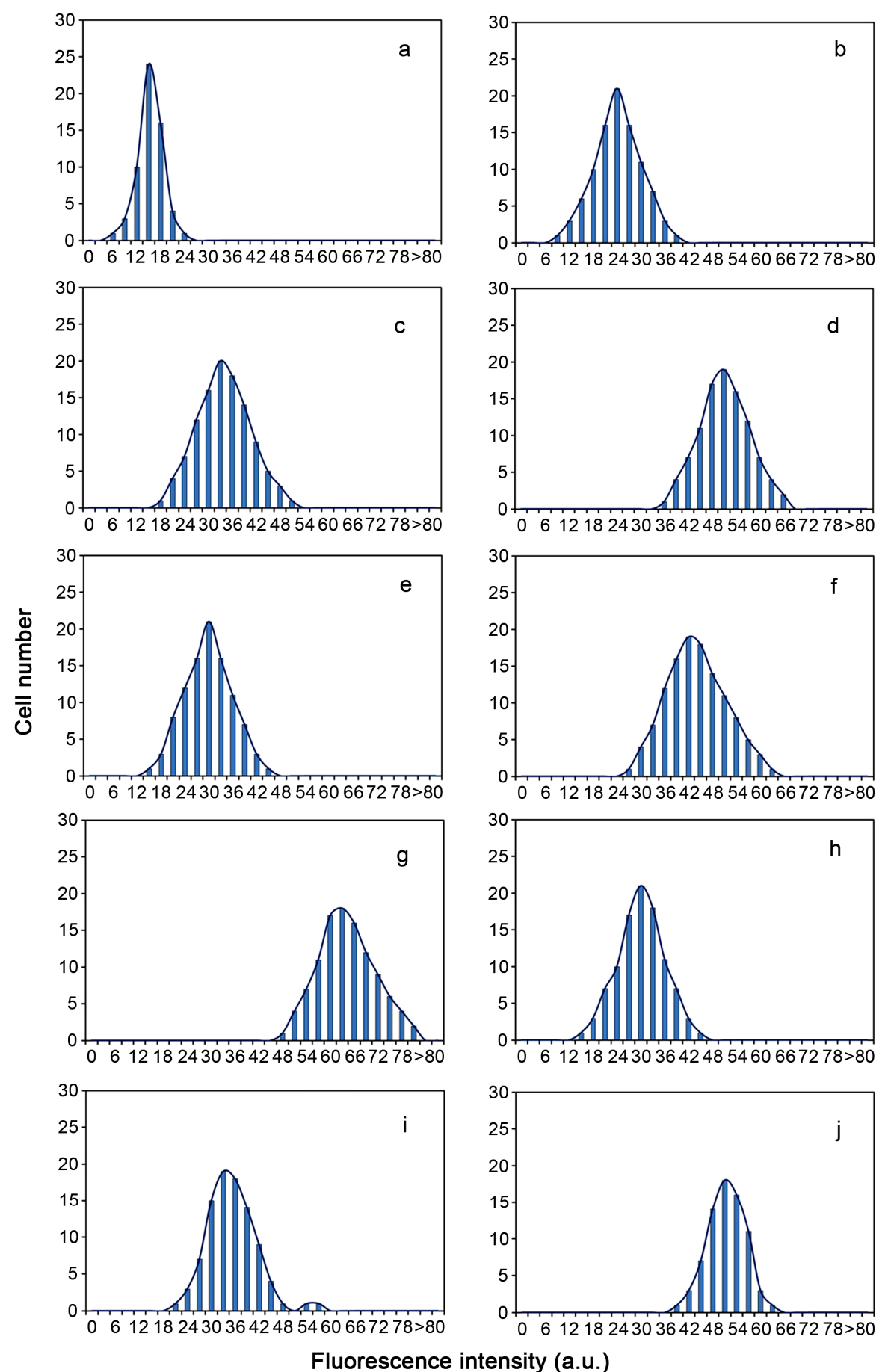

Figure 3. Static cytometric analyses, after LysoTracker Red fluorochromization, of THP-1 cell line as monocytic suspension (a) and after adhesion (PMA stimulation) to glass (b-d), to type I (e-g), and type IV (h-j), collagens. PMA concentration: (a) $0 \mathrm{nM}$; (b, e, h) $6 \mathrm{nM}$; (c, f, i) $30 \mathrm{nM}$; $(\mathrm{d}, \mathrm{g}, \mathrm{j}) 60 \mathrm{nM}$. All histograms are representative of one of three experiments.

it can regulate the quiescence or proliferation of some cells and stimulate their migration $[35,36]$. Some studies showed that modalities of interaction between collagen (solid or soluble) and cells are important: soluble type IV collagen added to the media stimulated by the migration of cells in vitro, indicating that cell responses to this molecule might be dependent upon the context in which the cells are exposed to collagen (i.e. assembled in the basement membrane, such a solid substrate or solubilized in the media). Furthermore, another study argued that 


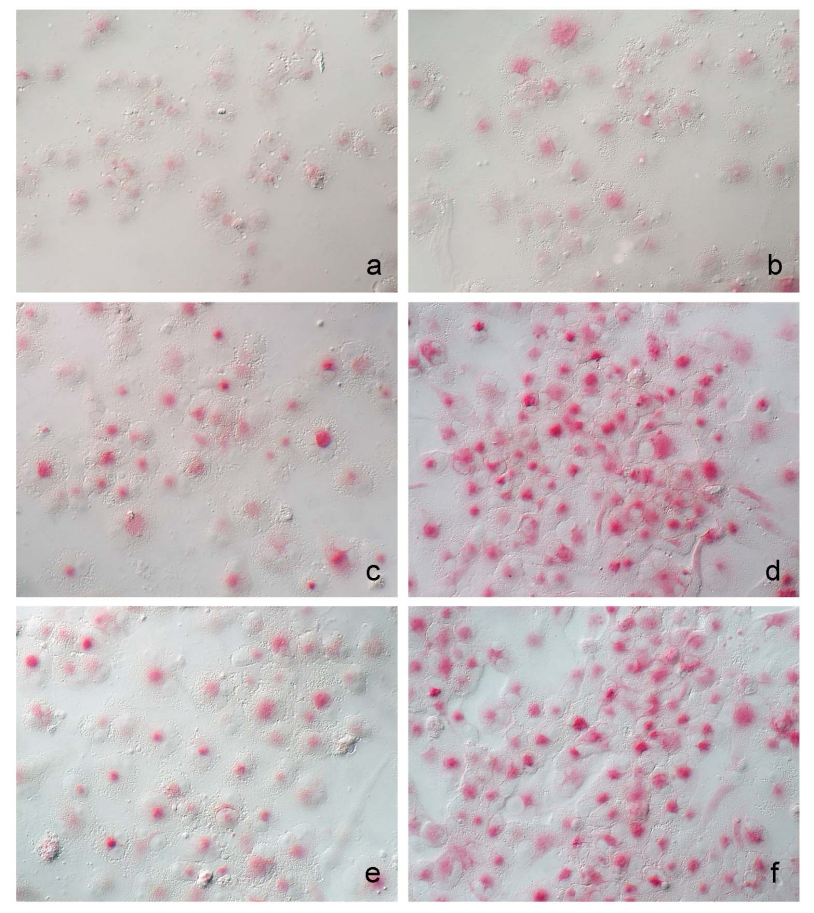

Figure 4. Acid phosphatase activity cytochemically demonstrated by azo-dye coupling method in THP-1 cells exposed to PMA (a, c, e): $6 \mathrm{nM}$; (b, d, f): $60 \mathrm{nM})$ and cultured on glass (a, b), type I collagen $(c, d)$, type IV collagen $(e, f)$. (objective $40 \times$, DIC contrast) Scale bar : $5 \mu$.

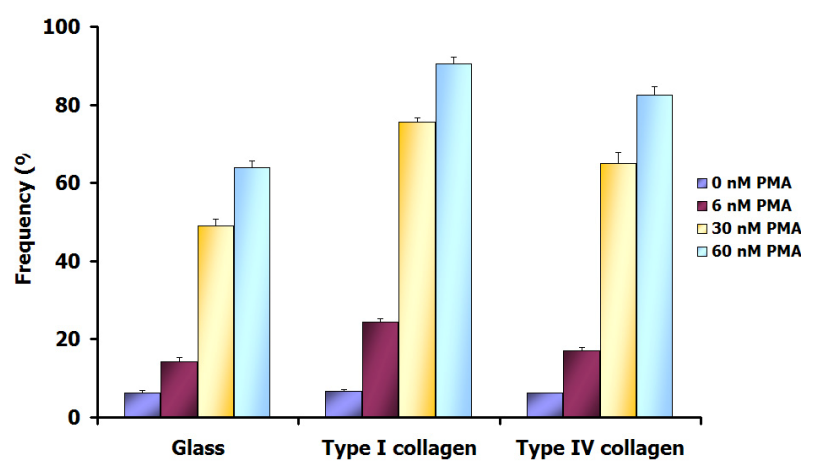

Figure 5. Frequency percent (mean \pm SEM) of cell positive for the cytochemical detection of acid phosphatase, in different experimental conditions. Statistical analysis was assessed by Kruskal-Wallis test in the different culture conditions by comparing the effects of PMA concentrations in the same cell adhesion substrate and the effects of the different substrate at the same PMA concentration. All results were significant with $p<0.05$, with the exception of the comparison between PMA-untreated cells.

smooth muscular cell chemotaxis towards type IV collagen was equal to or greater than that to type I collagen [36]. Thus, it is increasingly clear that cellular responses are governed not only by the type of collagen to which the cells are exposed, but also the concentration, conformation and context of the collagen molecules within which they are.
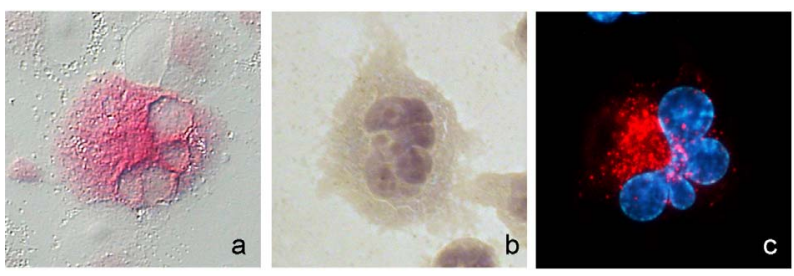

Figure 6. (a) High magnification view of a macrophagic cell (30 nM PMA, type I collagen) strongly positive for acid phosphatase cytochemically detectable. The red staining is mainly localized in the iuxta-nuclear region. (b) A similar cell with haematoxylyn-stained polylobed nucleus (objective 40x, DIC contrast in a). In (c), a cell with multilobulated nucleus (Hoechst 33342 staining) and evident LysoTracker Red fluorescent cytoplasmic spots is shown. Scale bar: $5 \mu$.

In our experimental model, in the transition from cells cultured on glass to those cultured on type I or IV collagen matrix, data have indicated a higher inductive effect toward monocyte-macrophage differentiation. This is in agreement with the evidence, in several tissues, of the role of extracellular matrix components (fibronectin, laminin, type I and IV collagens, etc.) in modulating in situ the processes of macrophagic differentiation and activation $[37,38]$. Our findings indicated that, between the two types of collagen, the higher effects on cytodifferentiation and activation are observed in the presence of type I collagen with respect to type IV collagen. In fact, after $60 \mathrm{nM}$ PMA and cell adhesion on type I collagen matrix, the greatest response to acid phosphatase reaction is reached, in terms of stained cell frequency and staining intensity. This confirms how some types of collagen may also affect other aspects correlated with differentiation and modulation of typical macrophage maturation-associated functions and the expression of specific enzyme activities. In general, these are changed during the differentiation of monocytes into macrophages. Peroxidase activity is lost, whereas nonspecific esterase activity remains constant [39].

In our work, we have intended to correlate the acquisition of specific cytokinetic features of THP-1 cells during PMA-induced differentiation with the acquisition of functional properties relative to lysosomal compartment evolution and the expression of AcP enzymatic activity, also in relation to the different features of adhesive substrate.

The quantification of lysosomal compartment was determined by means of cytofluorometric assays after LysoTracker Red fluorochromization, while the method of Barka \& Anderson [25] allowed us to analyze the lysosomal hydrolase acid phosphatase activity. The results demonstrated that the amount of lysosomes and the increase in specific lysosomal activity of THP-1 cells directly correlate with the degree of differentiation towards PMA-induced macrophage phenotype.

Interestingly, the acquisition of these cell properties 
appears to be dependent not only on the progressive cell enlargement related to differentiation level, but also influenced by the different characteristics of substrate adhesion. The results obtained by static cytometric analysis, after LT fluorochromization and the AcP assay, showed that ECM factors, such as collagens, are able to induce an increase in the amount and/or size of intact, endogenous lysosomes and a significant increase in hydrolase here considered. These findings are in agreement with some works [40-42] that demonstrated how the increase in differentiation of human monocyte was related to the production of other enzymes, such as the typical matrixdegrading enzyme matrix metalloproteinase. Furthermore, biochemical approaches on macrophagic activavtion effects by type I collagen in U937 cell line have demonstrated, beside an increase in acid phosphatase, a higher expression of esterase activity, thus underlying paracrin role of collagen [38], also in association with cytokine release [30].

The increase in acid phosphatase activity we demonstrated in THP-1 cells stimulated with the higher dose of PMA, beside expressing an increase in primary lysosome production by Golgi apparatus, in relation with macrophagic differentiation, can also express the presence of functioning lysosomes in both autophagic and heterophagic way [43]. The appearance of autophagosomes with PMA increasing dose, could be related to cytoplasmic damages deriving from cytotoxic effects of the substance [44]. Furthermore, hetero-phagosomes deriving from phagocytosis of apoptotic bodies as a consequence of programmed death events, that are relatively frequent after treatment, could also originate.

In conclusion, it is possible to underline that the differences emerging by comparing the various experimenttal conditions can be explained by considering the dependence on the PMA dose, which could generate various types of macrophages with a different level of differentiation. Moreover, the biological response, in terms of differenti- ate status modulation, could be modified in dependence on signals generated by culture conditions and adhesion in vitro.

\section{REFERENCES}

[1] Andreesen, R., Brugger, W., Scheinbenbogen, C., Kreutz, M., Leser, H.G., Rehm, A. and Löhr, G.W. (1990) Surface phenotype analysis of human monocyte to macrophage maturation. Journal of Leukocyte Biology, 47, 490497.

[2] Anegon, I., Blottiere, H., Cuturi, M.C., Blottiere, H., Cuturi, M.C., Lenne, Y., Trinchieri, G., Faust, J. and Perussia, B. (1993) Characterization of a human monocyte antigen, B148.4, regulated during cell differentiation and activation Journal of Leukocyte Biology, 53, 390-398.

[3] Huh, H.Y., Pearce, S.F., Yesner, L.M., Schindler, J.L. and Silverstein, R.L. (1996) Regulated expression of CD36 during monocyte-to-macrophage differentiation: potential role of CD36 in foam cell formation. Blood, 87, 2020-2028.

[4] Kreutz, M., Krause, S.W., Hennemann, B., Rehm, A. and Andreesen, R. (1992) Macrophage heterogeneity and differentiation: Defined serum-free culture conditions induce different types of macrophages in vitro. Research in Immunology, 143, 107-115. doi:10.1016/0923-2494(92)80087-2

[5] Prieto, J., Eklund, A. and Patarroyo, M. (1994) Regulated expression of integrins and other adhesion molecules during differentiation of monocytes into macrophages. Cellular Immunology, 156, 191-211. doi:10.1006/cimm.1994.1164

[6] Poli, G., Wang, J.M., Ruco, L., Rossini, S., Biondi, A., Mantovani, A. and Uccini, S. (1993) Expression and modulation of a mononuclear phagocyte differentiation antigen (PAM-1) during in vitro maturation of peripheral blood monocytes. International Journal of Clinical and Laboratory Research, 23, 83-87. doi:10.1007/BF02592288

[7] Kreutz, M., Andreesen, R., Krause, S.W., Szabo, A., Ritz, E. and Reichel, H. (1993) 1,25-dihydroxyvitamin D3 production and vitamin D3 receptor expression are developmentally regulated during differentiation of human monocytes into macrophages. Blood, 82, 1300-1307.

[8] Cleary, J.A., Kelly, G.E. and Husband, A.J. (1999) The effect of molecular weight and -1,6-linkages on priming of macrophage function in mice by $(1,3)$-D-glucan. Immunology and. Cell Biology, 77, 395-403. doi:10.1046/j.1440-1711.1999.00848.x

[9] Murao, S., Gemmell, M.A., Callaham, M.F., Anderson, N.L. and Huberman, E. (1983) Control of macrophage cell differentiation in human promyelocytic HL-60 leukemia cells by 1,25-dihydroxyvitamin D3 and phorbol12-myristate-13-acetate. Cancer Research, 43, 4989-4996.

[10] Olsson, I., Gullberg, U., Ivhed, I. and Nilsson, K. (1983) Induction of differentiation of the human histiocytic lymphoma cell line U-937 by 1 alpha,25-dihydroxycholecalciferol. Cancer Research, 43, 5862-5867.

[11] Fleit, H.B. and Kobasiuk, C.D. (1991) The human monocyte-like cell line THP-1 expresses Fc gamma RI and Fc gamma RII. Journal of Leukocyte Biology, 49, 556-565.

[12] Rovera, G., O’Brien, T.G. and Diamond, L. (1979) Induction of differentiation in human promyelocytic leukemia cells by tumor promoters. Science, 204, 868-870. doi:10.1126/science. 286421

[13] Kohro, T., Tanaka, T., Murakami, T., Wada, Y., Aburatani, H., Hamakubo, T., Kodama, T. (2004) A compareson of differences in the gene expression profiles of phorbol 12-myristate 13-acetate differentiated THP-1 cells and human monocyte-derived macrophage. Journal of Atherosclerosis and Thrombosis, 11, 88-97. doi:10.5551/jat.11.88

[14] Park, E.K., Jung, H.S., Yang, H.I., Yoo, M.C., Kim, C., et al. (2007) Optimized THP-1 differentiation is required for the detection of responses to weak stimuli. Inflammation Research, 56, 45-50. 
doi:10.1007/s00011-007-6115-5

[15] Schwende, H., Fitzke, E., Ambs, P. and Dieter, P. (1996) Differences in the state of differentiation of THP-1 cells induced by phorbol ester and 1,25-dihydroxyvitamin D3. Journal of Leukocyte Biology, 59, 555-561.

[16] Spano, A., Barni, S. and Sciola, L. (2013) PMA withdrawal in PMA-treated monocytic THP-1 cells and subsequent retinoic acid stimulation, modulate induction of apoptosis and appearance of dendritic cells. Cell Proliferation, 46, 328-347. doi:10.1111/cpr.12030

[17] Gantner, F., Kupferschmidt, R., Schudt, C., Wendel, A, Hatzelmann, A. (1997) In vitro differentiation of human monocytes to macrophages: Change of PDE profile and its relationship to suppression of tumour necrosis factoralpha release by PDE inhibitors. British Journal of Pharmacology, 121, 221-231. doi:10.1038/sj.bjp.0701124

[18] Spano, A., Monaco, G., Barni, S. and Sciola L. (2007) Expression of cell kinetics and death during monocytemacrophage differentiation: Effects of Actinomycin D and Vinblastine treatment. Histochemistry and Cell Biology, 127, 79-94. doi:10.1007/s00418-006-0227-9

[19] De Fougerolles, A.R. and Koteliansky, V.E. (2002) Regulation of monocyte gene expression by the extracellular matrix and its functional implications. Immunological Reviews, 186, 208-220. doi:10.1034/j.1600-065X.2002.18617.x

[20] Robinson, J.M. and Karnovsky, M.J. (1983) Ultrastructural localization of several phosphatases with cerium. Journal of Histochemistry and Cytochemistry, 31, 11971208. doi:10.1177/31.10.6309949

[21] Wiese, M., Berger, O., Stierhof, Y.D., Wolfram, M., Fuchs, M. and Overath, P. (1996) Gene cloning and cellular localization of a membrane-bound acid phosphatase of Leishmania mexicana. Molecular and Biochemical Parasitology, 82, 153-165. doi:10.1016/0166-6851(96)02729-6

[22] Karnovsky, M.L. and Lazdins, J.K. (1978) Biochemical criteria for activated macrophages. Journal of Immunology, 121, 809-813.

[23] Matsubara, S. (2002) Glucose-6-phosphate dehydrogenase and mouse Kupffer cell activation: An ultrastructural dual staining enzyme-cytochemical study. Histochemistry and Cell Biology, 118, 345-350.

[24] Campbell, G.R. and Spector, S.A. (2011) Hormonally active vitamin D3 (1alpha,25-dihydroxycholecalciferol) triggers autophagy in human macrophages that inhibits HIV-1 infection. Journal of Biological Chemistry, 286, 18890-18902. doi:10.1074/jbc.M110.206110

[25] Barka, T. and Anderson, P.J. (1962) Histochemical methods for acid phosphatase using hexazonium pararosanilin as coupler. Journal of Histochemistry and Cytochemistry, 10, 741-753. doi: $10.1177 / 10.6 .741$

[26] Jacob, S.S., Shastry, P. and Sudhakaran, P.R. (2002) Monocyte-macrophage differentiation in vitro: Modulation by extracellular matrix protein substratum. Molecular and Cellular Biochemistry, 233, 9-17. doi:10.1023/A:1015593232347

[27] Siljander, P.R., Hamaia, S., Peachey, A.R., Slatter, D.A., Smethurst, P.A., Ouwehand, W.H., Knight, C.G. and Far- ndale, R.W. (2004) Integrin activation state determines selectivity for novel recognition sites in fibrillar collagens. Journal of Biological Chemistry, 279, 47763-47772. doi:10.1074/jbc.M404685200

[28] Wang, P., Ballestrem, C. and Streuli, C.H. (2011) The C terminus of talin links integrins to cell cycle progression. Journal of Cell Biology, 195, 499-513. doi: $10.1083 /$ jcb. 201104128

[29] Assoian, R.K. and Schwartz, M.A. (2001) Coordinate signaling by integrins and receptor tyrosine kinases in the regulation of G1 phase cell-cycle progression. Current Opinion in Genetics \& Development, 11, 48-53. doi:10.1016/S0959-437X(00)00155-6

[30] Sondag, C.M. (2010) Adhesion of monocytes to type I collagen stimulates an APP-dependent proinflammatory signaling response and release of A $\beta 1-40$. Journal of Neuroinflammation, 7, 22-31. doi:10.1186/1742-2094-7-22

[31] Auwerx, J. (1991) The human leukemia cell line, THP-1: A multifacetted model for the study of monocyte-macrophage differentiation. Experientia, 47, 22-31. doi:10.1007/BF02041244

[32] Zhu, L. and Skoultchi, A.I. (2001) Coordinating cell proliferation and differentiation. Current Opinion in Genetics \& Development, 10, 91-97. doi:10.1016/S0959-437X(00)00162-3

[33] Hollenbeck, S.T., Itoh, H., Louie, O., Faries, P.L., Liu, B. and Kent, K.C. (2004) Type I collagen synergistically enhances PDGF-induced smooth muscle cell proliferation through pp60src-dependent crosstalk between the alpha2beta1 integrin and PDGFbeta receptor. Biochemical and Biophysical Research Communications, 325, 328337. doi:10.1152/ajpgi.00262.2003

[34] Wesley, R.B. 2nd, Meng, X., Godin, D. and Galis, Z.S. (1998) Extracellular matrix modulates macrophage functions characteristic to atheroma: Collagen type I enhances acquisition of resident macrophage traits by human peripheral blood monocytes in vitro. Arteriosclerosis, Thrombosis, and Vascular Biology, 18, 432-440. doi:10.1161/01.ATV.18.3.432

[35] Sanders, M.A. and Basson, M.D. (2004) Collagen IV regulates Caco-2 migration and ERK activation via $\alpha_{1} \beta_{1}$ and $\alpha_{2} \beta_{1}$-integrin-dependent Src kinase activation. American Journal of Physiology. Gastrointestinal and Liver Physiology, 286, G547-557. doi:10.1152/ajpgi.00262.2003

[36] Adiguzel, E., Ahmad P.J., Franco, C., Michelle, P. and Bendeck, M.P. (2009) Collagens in the progression and complications of Atherosclerosis. Vascular Medicine, 14, 73-89.

[37] Armstrong, J.W. and Chapes, S.K. (1994) Effects of extracellular matrix proteins on macrophages differentiation, growth and function: Comparison of liquid and agar culture system. Journal of Experimental Zoology, 269, 178187. doi:10.1002/jez.1402690303

[38] Matheson, L.A., Maksym, G.N., Santerre, J. P., Rosalind, S. and Labow, R.S. (2006) The functional response of U937 macrophage-like cells is modulated by extracellular matrix proteins and mechanical strain. Biochemistry and Cell Biology, 84, 763-773. 
doi:10.1139/o06-093

[39] Miranda, M.B., McGuire, T.F. and Johnson, D.E. (2002) Importance of MEK-1/-2 signaling in monocytic and granulocytic differentiation of myeloid cell lines. Leukemia, 16, 683-692. doi:10.1038/sj.leu.2402400

[40] Galt, S.W., Lindemann, S., Medd, D., Allen, L.L., Kraiss, L.W., Harris, E.S., Prescott, S.M., McIntyre, T.M., Weyrich, A.S. and Zimmerman, G.A. (2001) Differential regulation of matrix metalloproteinase- 9 by monocytes adherent to collagen and platelets. Circulation Research, 89, 509-516. doi:10.1161/hh1801.096339

[41] Shapiro, S.D., Kobayashi, D.K., Pentland, A.P. and Welgus, H.G. (1993) Induction of macrophage metalloproteinases by extracellular matrix. Evidence for enzymeand substratespecific responses involving prostaglandin- dependent mechanisms. Journal of Biological Chemistry, 268, 8170-8175.

[42] Lepidi, S., Kenagy, R.D., Raines, E.W., Chiu, E.S., Chait, A., Ross, R. and Clowes, A.W. (2001). MMP9 produc- tion by human monocyte-derived macrophages is decreased on polymerized type I collagen. Journal of Vascular Surgery, 34, 1111-1118. doi:10.1067/mva.2001.119401

[43] McDowell, E.M. (1973) Acid phosphatase activity in Golgi apparatus and related structures in pars recta of rat kidney studied by thin and semi-thin section cytochemistry. Histochemie, 34, 281-291. doi:10.1007/BF00306300

[44] Goel, G., Makkar, H.P.S., Francis, G. and Becker, K. (2007) Phorbol esters: Structure, biological activity, and toxicity in animals. International Journal of Toxicology, 26, 279-288. doi:10.1080/10915810701464641 